\title{
Relationship between communication skills training and doctors' perceptions of patient similarity
}

\author{
Claudine Clucas, Lindsay St Claire \\ Centre for Hearing and Balance Studies, University of Bristol, UK
}

Correspondence: Claudine Clucas, Centre for Hearing and Balance Studies, University of Bristol, UK

Email: c.clucas@ucl.ac.uk

\begin{abstract}
Objectives: The study's primary aim was to investigate whether participants who have received more communication skills training see patients as less similar to one another. The study's secondary aim was to explore differences in perceived patient similarity between male and female doctors, hospital doctors and general practitioners and medical students and doctors.

Methods: This study used a cross-sectional design. Thirtysix hospital consultants, 35 general practitioners and 56 clinical medical students in the United Kingdom were recruited via a snowballing technique and medical student societies. They completed a questionnaire in which they indicated hours and form of communication skills training received and rated perceptions of the two last patients they had seen on a 3 -item semantic differential scale. Data collection took place via the post, e-mail or the web. Pear-
\end{abstract}

son Chi-square and Kruskal-Wallis tests were used to analyse the ratings.

Results: Participants with greater communication skills training ( $\geq 30$ hours) perceived patients as less similar to each other $(\mathrm{M}=3.95, \mathrm{SD}=2.35)$ than participants with medium (between 10 and 30 hours, $\mathrm{M}=3.14, \mathrm{SD}=2.62$ ) and smaller communication skills training ( $\leq 10$ hours, $\mathrm{M}=$ $2.69, \mathrm{SD}=2.70)$, Kruskal-Wallis test $(2,119)=6.78, \mathrm{p}=.03$. There was no difference in perceived patient similarity according to the doctor's gender and place of work.

Conclusions: Communication skills training appears to decrease perceived similarity of patients but more research is needed to establish causality. Implications for patient satisfaction and doctors' respect for patients are discussed.

Keywords: Communication skills training, personalisation, general practitioners, hospital doctors, medical students

\section{Introduction}

Patients want doctors to show interest in them as people, which is likely to provide some relief from common feelings of vulnerability, depression, anxiety and uncertainty. ${ }^{1-5}$ However, patients often report being treated as non-persons or cases, especially when receiving inpatient care, and these experiences are a common reason for dissatisfaction..$^{6-8}$ Formal communication skills training (CST) with a patientcentred approach is being offered in undergraduate and postgraduate medical education in an attempt to improve the situation. CST is designed to encourage doctors to engage in a collaborative relationship with each patient, treating him/her as a whole person and focusing on his/her illness experience. ${ }^{9}$ Despite evidence to support its effectiveness in improving communication skills, ${ }^{10,11}$ it is unclear whether CST encourages healthcare professionals to perceive their patients as individuals.

Reasons for patients' experiences of being treated as non-persons or cases are not well understood. Aspects of current medical practice are likely to play a role. For example, according to Crossley, the use of medical technology encourages objectification of the patient. ${ }^{2}$ Similarly, a working party of The Royal College of General Practitioners states that 'assimilating medical science is a dehumanizing process, for it is achieved by breaking down human structures and functions into parts' (p. 160). ${ }^{12}$ There is evidence that medical students become more disease-focused as they progress through their clinical education. ${ }^{13}$ Another possible explanation for patients' experiences is based on Social 
Categorisation Theory. ${ }^{14}$ Doctor-patient relationships are intergroup as well as interpersonal; the doctor is expected to act as a professional expert as well as to build personal rapport with his/her patients. ${ }^{15-17}$ According to Social Categorisation Theory, cognitive effects of categorisation into groups include increased within group similarity. ${ }^{14}$ Doctors and medical students who act in terms of their medical professional identifications will tend to categorise patients as a relevant outgroup and thus tend to see patients as more similar to each other.

Semantic differential scales are commonly used to measure similarity in perceptions in psychology. ${ }^{18-20}$ A study by Fracchia and colleagues used semantic differential scales to measure depersonalisation of former mental patients. ${ }^{21} \mathrm{~A}$ lack of difference between the ratings for mildly ill, moderately ill and severely ill former mental patients on a semantic differential scale was interpreted as evidence of lack of individuation of former mental patients, with participants responding to the label or categorical classification rather than individual differences implicit in the various levels of severity.

Communication skills training should help redress this situation with its focus on treating the patient as a whole person and paying attention to the individual patient's illness experience. Greater amounts of CST should therefore be associated with greater personalisation of patients. CST might encourage doctors to move from interacting with patients at an intergroup level to interacting with them at an interpersonal level. Research supports that medical students and health care professionals engage in more patientcentred behaviours following CST. ${ }^{9,22,23}$ In particular, as a result of CST, primary care residents, general practitioners (GPs), physicians and cancer health professionals asked more open, more psychosocial and fewer closed questions, ${ }^{24-26}$ recognized more psychosocial behaviours in patients and clarified psychosocial concerns, ${ }^{11,27-29}$ explored health beliefs more, elicited more patients' concerns and opinions, ${ }^{26,30,31}$ invited patients to share decisions and negotiated about the process of health care ${ }^{31}$ and made more empathic responses ${ }^{32}$. Concerns that training effects are not maintained over time, ${ }^{33}$ or incorporated into practice ${ }^{34}$ are relatively few and evidence supporting them is mixed. ${ }^{33}$ Our primary aim is to investigate whether doctors and medical students who have received more CST will see patients as less similar to one another.

Research also supports differences in patientcentredness according to the doctor's gender and place of work. Female doctors tend to be more patient-centred than male doctors ${ }^{35}$ and there are indications that GPs hold more patient-centred norms than hospital doctors. ${ }^{36}$ Practicing doctors might be more likely than medical students to depersonalise patients given that they might be more likely to rely on mental categories they have developed with experience. Our secondary aim is to explore differences in perceived patient similarity between male and female doctors, between hospital doctors and GPs, and between medical students and practicing doctors.

\section{Methods}

\section{Sample}

Thirty-five GPs and 36 hospital doctors were recruited via a snowballing technique ${ }^{37}$ in cities across the United Kingdom (UK) in 2006/2007. To minimise possible biases arising as a result of participants choosing other participants similar to themselves, the sample was started with as many and as diverse doctors as possible. Thirty-six respondents were female and 35 were male. The registration year ranged from 1970 to 2005 (median 1994). Fifty-seven medical students in their clinical years were also recruited from three British medical schools (Bristol University, St George's University, Dundee University) via medical student societies. Twenty-two were in their fifth (final) year, 17 in their fourth year and 18 in their third year. Thirty-five were female and 22 were male.

\section{Materials and scoring}

A one page questionnaire comprised four sections:

Section 1 concerned CST: number of hours received in and since medical school, the form of CST received (the options of role play and feedback, lecture and discussion were presented and participants were asked to tick "yes" or "no" for each), whether or not they received CST with a patient-centred approach (defined as a focus on the illness experience, a collaborative relationship and tailoring treatment to each patient's unique needs) and the training guide used (the Calgary-Cambridge guide was suggested as an example and participants were asked to write which guide was used). Participants were asked to name the guide used to get an indication of their level of CST interest.

Amount of CST could not be treated as continuous variable based on the number of hours reported because several doctors and medical students provided a range for the number of hours received (e.g. "between 20 and 30" or " $<10$ "). For this reason, participants were categorized as "smaller" ( $\leq 10 \mathrm{hrs})$, "medium" (10-30 hrs) and "greater" ( $\geq 30 \mathrm{hrs}$ ) amounts of CST training based on the number of CST hours in and since medical school reported (approximately one third of participants fell into each category). Participants who gave an estimate of the number of hours received were subsequently placed in these categories (see Table 1).

Section 2 concerned perceived similarity of patients: participants were asked to rate the two last patients they had seen (having a similar medical condition) using a three-item semantic differential scale. These scales are widely used in social psychology and have been shown to have high validity and reliability. ${ }^{18,38}$ The items were good-bad, activepassive and strong-weak presented in counterbalanced order and each scored from -3 to +3 with scores reverse 
coded such that a higher score indicated a more negative perception of the patient. These adjectives were selected because they have been shown to have strong loadings on the evaluative, activity and potency dimensions underlying semantic differential ratings. ${ }^{18}$ Participants were asked to rate the two last patients they had seen with a similar medical condition to reduce variability of ratings. Participants only rated one pair of patients to keep the questionnaire short in order to increase the response rate, and also to avoid focusing the participants' attention on our interest in their perceptions of patient similarity.

Table 1. Perceived amount of communication skills' training according to status in the UK $(n=120)$

\begin{tabular}{lccccccc}
\hline \multirow{2}{*}{ Group } & \multicolumn{2}{c}{$\begin{array}{c}\text { None or } a \\
\text { little }(\leq 10)\end{array}$} & Moderate & \multicolumn{2}{c}{$\begin{array}{c}\text { A lot } \\
(\geq 30)\end{array}$} & \multirow{2}{*}{ Total } \\
\cline { 2 - 6 } & $\mathrm{N}$ & $\%$ & $\mathrm{~N}$ & $\%$ & $\mathrm{~N}$ & $\%$ & \\
\hline Whole sample & 33 & 27.5 & 42 & 35 & 45 & 37.5 & $120^{*}$ \\
Hospital doctors & 19 & 54.3 & 8 & 22.9 & 8 & 22.9 & 35 \\
General Practitioners & 6 & 18.8 & 14 & 43.8 & 12 & 37.5 & 32 \\
Medical students & 8 & 15.1 & 20 & 37.7 & 25 & 47.2 & 53 \\
\hline
\end{tabular}

*8 participants could not be assigned to any categories of CST because they did not provide sufficient information

Semantic distance was calculated by summing the differences between scores of the two patients over each of the bipolar adjectives of the semantic differential scale. The three items loaded strongly on one factor in a principal components analysis (loadings >.65), supporting the unidimensional structure of the measure, and Cronbach's a was .66. Scores could range from 0 to 18 , with lower scores indicating perceived similarity.

Questions about the gender, approximate age and nationality of the patients were also asked to measure the demographic similarity of patients. A categorical variable, demographic patient similarity, was created based on the 3 variables of age (patients aged within ten years of each other were judged to be of similar age), sex and nationality: $1=$ high (similar/same for all three characteristics), 2 = medium (different on one characteristic) and $3=$ low (different on two or three characteristics).

Section 3 comprised two 5-point Likert scales which measured the strength of participants' medical professional identification. These were scored from 1 (agree not at all) to 5 (agree extremely): 'I feel like I belong in the medical world' and 'I am pleased to be a doctor' (or pleased to soon be a doctor for medical students) (adapted from Doosje and colleagues ${ }^{39}$ ), Cronbach's a was 0.81 .

Section 4 comprised demographic questions: gender, nationality, year of registration (or year of medical school for the medical students) and whether working in general practice or a hospital.

\section{Data Collection}

Ethical permission was granted by the NHS Central Office for Research Ethics Committees and Research and Development approval from relevant sites was obtained. Doctors received a paper version of the questionnaire with a stamped addressed envelope from another doctor or an electronic copy of the questionnaire via e-mail. The questionnaires were either returned in the provided stamped addressed envelopes or by e-mail. Among doctors who received a paper version of the questionnaire, the response rate was $54 \%$. The questionnaires were e-mailed to medical students or a web link to online versions was sent to medical students by medical student societies. Medical students returned the questionnaire by e-mail or completed it online. For the web based survey, special online survey settings were selected to prevent multiple responses from a single individual and to prevent results from being made available to the public in order to preserve confidentiality.

\section{Statistical analyses}

Pearson Chi-square tests were used to investigate associations between categorical variables and Kruskal-Wallis tests were used to investigate associations between categorical and continuous variables that were not normally distributed.

\section{Results}

All the medical students received CST with role play, discussion and lecture. The vast majority of doctors received CST with role play (90.1\%). Among doctors and medical students who received CST (31 hospital doctors, 34 GPs, 57 medical students), 15 hospital doctors (48.4\%), 29 GPs (85.3\%) and 55 medical students (96.5\%) reported that they received CST with a patient-centred approach and 8 hospital doctors (25.8\%), 18 GPs (52.9\%) and 35 medical students $(61.4 \%)$ gave the name of the training guide used.

A Kruskal-Wallis test showed a significant association between reported amount of CST and perceived similarity of patients, KW-test $(2,119)=6.78, \mathrm{p}=.034[d$ (smaller to greater training) $=0.5$ ]. Doctors and medical students who received smaller amounts of CST perceived patients as more similar to one another $(\mathrm{M}=2.69, \mathrm{SD}=2.70)$ than doctors and medical students with medium CST $(\mathrm{M}=3.14, \mathrm{SD}=$ 2.62) and doctors and medical students with greater CST $(\mathrm{M}=3.95, \mathrm{SD}=2.35)$. Further Kruskal-Wallis tests did not show a significant relationship between medical school or year of medical school $\left(3^{\text {rd }}\right.$ vs. $4^{\text {th }}$ vs. $\left.5^{\text {th }}\right)$ and perceived similarity of patients.

Analyses were conducted to identify any potential covariates. Chi-square tests revealed significant associations between reported amount of CST and status (hospital doctor vs. GP vs. medical student), $\mathrm{X}^{2}(4,120)=18.60, \mathrm{p}=$ .001 , with hospital doctors reporting less CST than GPs and medical students (see Table 1) and between reported amount of CST and gender, $\mathrm{X}^{2}(2,120)=7.24, \mathrm{p}=.027$, with males reporting less CST than females (13 (24.5\%) males reported $\geq 30$ hrs vs. 32 ( $47.8 \%$ ) females). However, neither gender nor status was significantly associated with perceived similarity of patients at $\mathrm{p}<.25$. There was no 
association between reported amount of CST and demographic patient similarity. Awareness of the nature of CST as patient-centred and of the training guide used were not significantly associated with perceived similarity of patients at $\mathrm{p}<.25$.

A Kruskal-Wallis test also showed a significant relationship between year of registration and reported amount of CST with doctors with smaller and medium CST having registered earlier $(\mathrm{M}$ (smaller $)=1989.21, \mathrm{SD}=9.16 ; M$ $($ medium $)=1990.77, \mathrm{SD}=9.16)$ than doctors with greater CST $(\mathrm{M}=1997.5, \mathrm{SD}=8.64), \mathrm{KW}$-test $(2,66)=11.96, \mathrm{p}=$ .003 . However, year of registration was not significantly associated with perceived similarity of patients at $\mathrm{p}<.25$.

A borderline relationship was found between strength of medical professional identification (high/low) and perceived patient similarity, MW-U $(1,127)=1550, \mathrm{p}=.082$, which further validates our measure of perceived patient similarity. Participants with high strength of medical professional identification perceived patients as more similar $(\mathrm{M}=3.07, \mathrm{SD}=2.58)$ than participants with low strength of medical professional identification $(\mathrm{M}=3.87$, $S D=2.61$ ). The relationship between reported amount of CST and strength of medical professional identification was not significant at $\mathrm{p}<.25$.

\section{Discussion}

Analyses supported our hypothesis that doctors and medical students who have received more CST will see patients as less similar to one another. The findings suggest that patients could be more likely to report being treated as individuals by doctors who have received CST, and as a result be more satisfied since previous research has shown that patients want to be treated as individuals and not as cases. This is important given that doctors are increasingly likely to receive CST in medical school ${ }^{40}$ and since patient satisfaction is associated with greater use of services, adherence to treatment and fewer malpractice lawsuits. ${ }^{41,42}$ More research is however needed to support the premise that personalisation of patients results in treating patients as individuals. Another key implication of the findings is that the practice of patient-centred communication could lead to doctors developing an attitude of respect towards patients since such individualized attitude involves a positive evaluation of the person as valuable and deserving of careful attention and proper consideration. ${ }^{43}$ Patients who feel respected are more likely to adhere to the doctor's advice and revisit the doctor, perceive themselves as less ill and experience higher self-esteem. ${ }^{44}$

No differences were found in terms of perceived patient similarity according to the doctor's gender, place of work or status as practising doctor or medical student. The lack of a difference in perceived patient similarity between hospital doctors and GPs is surprising since hospital doctors reported less CST. It is possible that a much bigger difference in amount of CST is needed to affect perceived similarity of patients. Indeed, a larger difference in perceived patient similarity was found between practising doctors and students, although this did not reach significance.

The findings that doctors who registered earlier reported less CST than doctors who registered later and that hospital doctors reported less CST than GPs and medical students is consistent with the literature. Indeed, despite evidence that patient-centred communication skills can be effectively taught and learned, it is only in the last twenty years that CST became an integral part of the medical curriculum $^{40,45}$ and its implementation is inconsistent across branches of medicine, occupying a more important role in GP vocational training compared to postgraduate specialist training. ${ }^{46}$ It is also possible that hospital doctors reported receiving less CST because they paid less attention to the training; patient-centred communication skills are often taught by psychologists, GPs or psychiatrists instead of other hospital doctors, ${ }^{8,9}$ making it less likely that patientcentred values be perceived as normative for hospital doctors. Hospital doctors were also less likely to report that the CST received used a patient-centred approach, and to be aware of the training guide used, compared to GPs and medical students, even after adjusting for amount of CST received.

Our measure of perceived patient similarity was intended to measure depersonalisation of patients. Demand characteristics should have been reduced by using this measure since doctors were unaware that we were interested in their perceptions of patient similarity. There were four limitations to the study. First, the reported amount of CST was a naturally occurring variable and therefore a causal effect of perceived CST on perceived similarity of patients cannot be inferred. However, a relationship between CST and perceived similarity of patients was also found for medical school students who do not have much choice in deciding how many hours of CST they receive, which reinforced the possibility of a causal relationship between CST and perceived similarity of patients. Further research could investigate this using a randomized controlled trial in which doctors are randomly assigned to different amounts of CST and their perceived similarity of patients measured at the end of the intervention. Second, the study measured reported amounts of CST instead of actual CST and research is needed to confirm that these correspond. Third, to the best of our knowledge, there are no clear guidelines as to how many hours of CST are necessary and sufficient. Research should investigate whether there is a number of hours of CST after which no significant further benefit is gained. Such information would be very helpful for medical education and could save costs to providers of CST. Fourth, a snowballing sampling technique was used to recruit the doctors and one cannot be sure that the participants are representative of the general doctor population. Among doctors who received a paper version of the questionnaire, the response rate was only $54 \%$; this is however reasonable 
given the well-known documented difficulties in getting doctors to complete and return questionnaires. ${ }^{47}$ There is also possibility of a self-selection bias, although it should have been similar in the different groups and is unlikely to have affected the validity of the findings.

\section{Acknowledgments}

We acknowledge assistance from Dr Michael Whitfield, Dr Kyra Arnott, Dr Mary Welsh, Dr Chris Griffiths, Dr David DeBerker and Ms Jenny Symonds for their help in recruiting doctors and the medical student societies "the Galanicals", "Medsin Society" and "Medsin" for their help in recruiting medical students.

\section{References}

1. Lupton D. Consumerism, reflexivity and medical encounter. Soc Sci Med. 1997;43:373-81.

2. Crossley ML. Rethinking health psychology. Buckingham: Open University Press; 2000.

3. Davey B, Seale C. Experiencing and explaining disease. 3rd ed. Buckingham: Open University Press; 2002.

4. Lupton D. Medicine as Culture: Illness, disease and the body in western societies. 2nd ed. London: Sage; 2003.

5. Leigh H, Reiser MF. The patient: biological, psychological and social dimensions of medical practice. New York: Plenum Publishing Corporation; 1980.

6. Kumar P, Clark M. Clinical medicine. 6th ed. Spain: Elsevier (Saunders) Limited; 2005.

7. Coyle J. Exploring the meaning of 'dissatisfaction' with health care: the importance of 'personal identity threat'. Sociol Health Illn. 1999;21:95-124.

8. Sinclair S. Making doctors: An institutional apprenticeship. Oxford: Berg; 1997.

9. Silverman J, Kurtz S, Draper J. Skills for communicating with patients. 2nd Ed. Oxon: Radcliffe Publishing Ltd; 2005. 10. Maguire $\mathrm{P}$, Roe $\mathrm{P}$, Goldberg $\mathrm{D}$, Jones S, Hyde C, O'Dowd T. The value of feedback in teaching interviewing skills to medical students. Psychol Med. 1978;8:695-704.

11. Hulsman RL, Ross WJG, Winnubst JAM, Bensing JM. Teaching clinically experienced physicians communication skills, a review of evaluation studies. Med Educ. 1999; 33:655-68.

12. The Royal College of General Practitioners. The future general practitioner. London: BMJ; 1972.

13. Wahlqvist $M$, Mattsson $B$, Dahlgren G, HartwigEricsson M, Henriques B, Hamark B, et al. Instrumental strategy: a stage in students' consultation skills training? Scand J Prim Health Care. 2005;23:164-70.

14. Tajfel, H. Cognitive aspects of prejudice. J Soc Psychol. 1969;25:79-97.

15. Turner JC, Hogg MA, Oakes PJ, Reicher SD, Wetherell MS. Rediscovering the social group: a self-categorization theory. New York: Basil Blackwell Ltd; 1987.

16. Tajfel H. Human groups and social categories: studies in social psychology. Cambridge: Cambridge University Press;
1981.

17. Watson B, Gallois C. Nurturing communication by health professionals towards patients: a communication accommodation theory approach. Health Commun. 1998;4:343-55.

18. Osgood Suci GT, Tannenbaum PH. The measurement of meaning. Urbana: University of Illinois; 1957.

19. Paluszny M, Beit-Hallahmi B. An assessment of monozygotic twin relationships by the semantic differential. Arch Gen Psychiatry. 1974;31:110-2.

20. St. Claire L, Clucas C. In sickness and in health: Influences of social categorizations on health-related outcomes. In: Jetten, J, Haslam, C, Haslam, S. A, editors. The social cure: Identity, health, and well-being. UK: Psychology Press; 2011.

21. Fracchia J, Sheppard C, Canale D, Ruest E, Cambria E, Merlis S. Community perception of illness levels of former mental patients: a failure to discriminate. Compr Psychiatry. 1976; 17:775-8.

22. Yedidia MJ, Gillespie CC, Kachur E, Schwartz MD, Ockene J, Chepaitis AE, et al. Effect of communication training on medical student performance. JAMA. 2003;290:1157-65.

23. Evans BJ, Sweet B, Coman GJ. Behavioural assessment of the effectiveness of a communication programme for medical students. Med Educ. 1993;27:344-50.

24. Jenkins, V, Fallowfield L. Can communication skills training alter physicians' beliefs and behavior in clinics? J Clin Oncol. 2002; 20:765-69.

25. Roter DL, Larson S, Shinitzky H, Chernoff R, Serwint JR, Adamo G, et al. Use of an innovative video feedback technique to enhance communication skills training. Med Educ. 2004;38:145-57.

26. Levinson W, Roter D. The effects of two continuing medical education programmes on communication skills of practicing primary care physicians. J Gen Intern Med. 1993;8:318-24.

27. Van Dulmen AM, Holl RA. Effects of continuing paediatric education in interpersonal communication skills. Eur J Pediatr. 2000; 159:489-95.

28. Maguire P, Faulkner A, Booth K, Elliott C, Hillier V. Helping cancer patients disclose their concerns. Eur J Cancer. 1996;32:78-81.

29. Roter, DL, Hall JA, Kern DE, Barker LR, Cole KA, Roca RP. Improving physicians' interviewing skills and reducing patients' emotional distress. A randomized clinical trial. Arch Intern Med. 1995;155:1877-84.

30. Joos SK, Hickam DH, Gordon GH, Baker LH. Effects of a physician intervention on patient care outcomes. J Gen Intern Med. 1996;11:147-55.

31. Langewitz WA, Eich P, Kiss A, Wossmer B. Improving communication skills--a randomized controlled behaviour ally oriented intervention study for residents in internal medicine. Psychosom Med. 1998;60:268-76.

32. Bonvicini KA, Perlin MJ, Bylund CL, Carroll G, Rouse 
RA, Goldstein MG. Impact of communication training on physician expression of empathy in patient encounters. Patient Educ Couns. 2009;75:3-10.

33. Gysels M, Richardson A, Higginson IJ. Communication training for health professionals who care for patients with cancer: a systematic review of effectiveness. Support Care Cancer. 2004;12:692-700.

34. Hoppe RB, Farquhar LJ, Henry R, Stoffelmayr B. Residents' attitudes towards and skills in counselling: using undetected standardized patients. J Gen Intern Med. 1990;5:415-20.

35. Roter DL, Hall JA, Aoki Y. Physician gender effects in medical communication: a meta-analytic review, JAMA. 2002;288:756-64.

36. Helman CG. Culture, health and illness. London: Butterworth Heineman; 2001.

37. Atkinson R, Flint J. Accessing hidden and hard-to-reach populations: snowball research strategies. Social research update; 2001 [cited 2010 October 9]; Available from: http://sru.soc.surrey.ac.uk/SRU33.html.

38. Eagly AH, Chaiken S. The psychology of attitudes. CA, USA: Wadsworth; 1993.

39. Doosje B, Ellemers N, Spears R. Perceived intragroup variability as a function of group status and identification. $J$ Exp Psychol. 1995;31:410-36.

40. Hargie O, Dickson D, Booran N, Hughes K. A Survey of Communication skills training in the U.K schools of medicine: present practices and prospective Proposals. Med
Educ. 1998; 32:25-37.

41. Stelfox HT, Gandhi TK, Orav JE, Gustafson ML. The relation of patient satisfaction with complaints against physicians and malpractice lawsuits. Am J Med. 2005,118:1126-33.

42. Keith RA. Patient satisfaction and rehabilitation services. Arch Phys Med Rehabil. 1998;79:1122-8.

43. Dillon R. Respect. The Stanford Encyclopaedia of Philosophy, Edward N. Zalta (ed.); 2010 [cited 2010 October 9]; Available from: http://plato.stanford.edu/archives/fall2010/entries/respect. 44. Clucas C, St Claire L. The effect of feeling respected and the patient role on patient outcomes. Applied Psychology: Health and Well-being. 2010;2:298-322.

45. Laidlaw ST, Kaufman DM, MacLeod H, van Zanten S, Simpson D, Wrixon W. Relationship of resident characteristics, attitudes, prior training and clinical knowledge to communication skills performance. Med Educ. 2006;40:1825.

46. Cooper V, Hassell A. Teaching consultation skills in higher specialist training: experience of a workshop for specialist registrars in rheumatology. Rheumatol. 2002;41:1168-71.

47. Kaner EF, Haighton CA, McAvoy BR. So much post, so busy with practice--so, no time! A telephone survey of general practitioners' reasons for not participating in postal questionnaire surveys. Br J Gen Pract. 1998;48:1067-69. 